\title{
A stepwise mutagenesis approach using histidine and acidic amino acid to engineer highly $\mathrm{pH}$-dependent protein switches
}

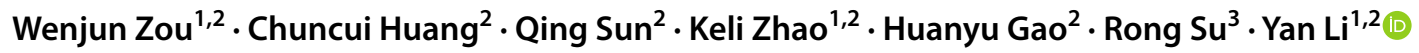

Received: 25 July 2021 / Accepted: 26 November 2021 / Published online: 20 December 2021

(c) King Abdulaziz City for Science and Technology 2021

\begin{abstract}
Antibody-based drugs can be highly toxic, because they target normal tissue as well as tumor tissue. The pH value of the extracellular microenvironments around tumor tissues is lower than that of normal tissues. Therefore, antibodies that engage in $\mathrm{pH}$-dependent binding at slightly acidic $\mathrm{pH}$ are crucial for improving the safety of antibody-based drugs. Thus, we implemented a stepwise mutagenesis approach to engineering $\mathrm{pH}$-dependent antibodies capable of selective binding in the acidic microenvironment in this study. The first step involved single-residue histidine scanning mutagenesis of the antibody's complementarity-determining regions to prescreen for $\mathrm{pH}$-dependent mutants and identify ionizable sensitive hotspot residues that could be substituted by acidic amino acids to obtain $\mathrm{pH}$-dependent antibodies. The second step involved single-acidic amino acid residue substitutions of the identified residues and the assessment of pH-dependent binding. We identified six ionizable sensitive hot-spot residues using single-histidine scanning mutagenesis. Nine pH-dependent antibodies were isolated using single-acidic amino acid residue mutagenesis at the six hot-spot residue positions. Relative to wild-type anti-CEA chimera antibody, the binding selectivity of the best performing mutant was improved by approximately 32-fold according to ELISA and by tenfold according to FACS assay. The mutant had a high affinity in the pH range of 5.5-6.0. This study supports the development of $\mathrm{pH}$-dependent protein switches and increases our understanding of the role of ionizable residues in protein interfaces. The stepwise mutagenesis approach is rapid, general, and robust and is expected to produce $\mathrm{pH}$-sensitive protein affinity reagents for various applications.
\end{abstract}

Keywords Antibody engineering $\cdot$ Carcinoembryonic antigen $\cdot$ Hot-spot residues $\cdot$ Tumor microenvironments

\section{Introduction}

Antibody-based anticancer therapy targets tumor-associated antigens that are highly expressed on tumor cells. However, these antigens are also present, to some degree, on the surface of somatic cells (Slaga et al. 2018; Szot et al. 2018).
Therefore, antibody treatments target both normal and tumor tissues, thereby increasing the risk of off-target toxicity to normal tissues (Haraya et al. 2019). As a result, there is a considerable need to improve tumor targeting and reduce the off-target toxicity of antibody drugs.

Rong Su

fssurong@163.com

1 College of Life Sciences, University of Chinese Academy of Sciences, Beijing 100049, China

2 Key Laboratory of Interdisciplinary Research, Institute of Biophysics, Chinese Academy of Sciences, Beijing 100101, China

Qing Sun

sunqing@ibp.ac.cn

Keli Zhao

zhaokeli@ibp.ac.cn

3 Department of Clinical Laboratory, Foshan Hospital of Traditional Chinese Medicine, Foshan 528000, Guangdong, China

Huanyu Gao gaohuanyu@moon.ibp.ac.cn 
The unique tumor microenvironment (TME) differences of tumor tissue compared with normal tissue (Kroemer and Pouyssegur, 2008; Vaupel 2010), such as lower oxygen levels (Ward et al. 2013), higher pressure (Ariffin et al. 2014), and a lower pH (Anderson et al. 2016; Cruz-Monserrate et al. 2014; Zhang et al. 2010), offer the opportunity to improve target selectivity. A growing body of literature demonstrates the acidic external microenvironment of tumor cells; this occurs because the glycolytic metabolism of tumors differs from that of normal tissue, and tumor cells secrete high levels of lactic acid (Warburg 1925). Recently, Rohani et al. demonstrated that malignant tumors are acidic, but not all cells found within a tumor are malignant cells. The extracellular $\mathrm{pH}$ of tumors ranges from 5.8 to 6.5 , compared with 7.2-7.4 for normal tissue (Gerweck and Seetharaman 1996; Rohani et al. 2019; Sulea et al. 2020). Consequently, pHdependent antibodies can be engineered that selectively bind in the acidic extracellular environment of tumors, thereby improving target selectivity and reducing off-target antibody toxicity.

Conceptually, two types of $\mathrm{pH}$-dependent antibodies with different functions can be obtained - either selectively weakening the binding affinity of wild-type antibodies at physiological $\mathrm{pH}(\mathrm{pH} 7.4)$ or introducing lower affinity at acidic $\mathrm{pH}$ (pH 6.0) (Igawa et al. 2014; Sulea et al. 2020). The engineering of $\mathrm{pH}$-dependent antibodies has overwhelmingly focused on weakening the binding at acidic $\mathrm{pH}$. These antibodies bind to overexpressed antigens, and the antibody-antigen complexes are dissociated in acidic endosomes; the antigens then undergo lysosomal degradation. Related studies have also focused on improving pharmacokinetics (Hong et al. 2021; Traxlmayr et al. 2014). In contrast, engineered $\mathrm{pH}$-dependent antibodies that selectively bind at acidic $\mathrm{pH}$ (i.e., they have weakened binding affinity at physiological $\mathrm{pH}$ ) have rarely been studied. To our knowledge, only two previous studies reported successful prospective engineering of $\mathrm{pH}$-dependent antibodies that had weakened binding at physiological $\mathrm{pH}$ and improved tumor-targeting capability. One study used a histidine scanning mutagenesis strategy for $\mathrm{pH}$ selectivity optimization based on computational design. However, the potential limitations of this approach are that 1 ) the potential sequence space for mutating proteins is restricted to histidine substitution; and 2) the possibility that non-histidine residues contribute to the $\mathrm{pH}$ sensitivity of binding is ignored (Gera et al. 2012).

Another study described a novel $\mathrm{H}+$ ion-dependent mechanism that blocks the antibody from binding to the antigen under normal conditions through the involvement of noncovalently bound physiological chemical(s) (e.g., bicarbonate or hydrogen sulfide). This reduces binding under normal physiological conditions while maintaining binding to the tumor (Chang et al. 2021). In that study, deep mutational scanning of the CDR region was used to screen $\mathrm{pH}$-dependent antibodies. All residues of the CDRs were included in a mutational study in which a single mutation was used as the mutation site and all 20 amino acids were applied as mutation parameters. However, deep mutational scanning can be a labor-intensive route for antibody engineering. Consequently, the antibody engineering strategy for $\mathrm{pH}$ dependence requires further exploration and improvement. Here, we designed a relatively simple stepwise mutagenesis approach based on ionizable groups-histidine and acidic amino acids (aspartic acid and glutamic acid) - to engineer $\mathrm{pH}$-dependent antibodies with selective binding in the acidic microenvironment.

The $\mathrm{pH}$ response stems from the reversible protonation and deprotonation of ionizable groups at the molecular level (Bazban-Shotorbani et al. 2017). Typical ionizable moieties include amines and carboxylic acids, which can be protonated or deprotonated at different $\mathrm{pH}$ values. In addition to histidine, ionizable groups are also found in the side chains of acidic amino acids, because they contain carboxylic acids, which can theoretically cause $\mathrm{pH}$ dependent conversion (Tang et al. 2019). Previous reports have indicated that acidic amino acids play an essential role in pH-dependent binding (Roche et al. 2006). Warwicker et al. recently demonstrated that the SARS-CoV-2 spike protein trimer structures undergo a $\mathrm{pH}$-dependent switch at acidic $\mathrm{pH}$ and that this involves E191, D985, and D398 acidic residues; this is thought to avoid immune surveillance of the open form of the receptor-binding domain of the S protein (Warwicker, 2021). Kumar et al. demonstrated that the ionization state of the acidic residues at the active site of hexokinase $\mathrm{B}$ were responsible for the opening and closing of the cleft between the two domains at different $\mathrm{pH}$ ranges, thereby affecting the structure and function of the enzyme (Kumar et al. 2004). In the current study, we applied acidic amino acid residue mutagenesis at ionizable sensitive hot-spot residues on the antibody surface as a logical approach to realize a $\mathrm{pH}$-dependent design.

As is well known, the engineering methods of $\mathrm{pH}$ dependent proteins usually identify the substitution position in the hot-spot residues as a starting point (Bonvin et al. 2015; Rötzschke et al. 2002). As a result, effectively identifying which hot-spot residues of antibody complexes are candidate mutagenesis sites is critical for introducing $\mathrm{pH}$-dependent interactions. However, it can be challenging to determine which residues are suitable for replacement by ionizable groups (e.g., aspartic acid and glutamic acid) to screen $\mathrm{pH}$-dependent antibodies. Previous efforts to identify candidate mutagenesis sites have all used computational structure-based design to guide the insertion of ionizable groups for screening $\mathrm{pH}$-dependent antibodies (Sarkar et al. 2002; Strauch et al. 2014; Sulea et al. 2020). However, this strategy is limited by the high cost of the 
experimental equipment; furthermore, it is not a general approach (Liu et al. 2018).

Here, we propose that single-histidine scanning can be used to identify candidate mutagenesis sites for inserting acidic amino acids and introducing $\mathrm{pH}$ dependence. Therefore, we employed the single-residue histidine scanning mutagenesis as the first step of a stepwise mutagenesis approach. It is worth mentioning that the single-histidine scanning approach differs from the conventional approach of the alanine scanning approach (Cunningham et al. 1990; Cunningham and Wells, 1989). Single-histidine scanning mutagenesis can not only predict whether the side chain of a specific amino acid residue plays a role in the antigen-antibody binding but also introduce a new function of identified ionizable sensitive epitope residues (Gera et al. 2012; Murtaugh et al. 2011) to identify suitable chargeable amino acid insertion sites. In addition, we used the Dual-pH capture ELISA to perform singleresidue histidine scanning mutagenesis. There are two purposes here. The first is to screen ionizable sensitive hot-spots through decreased binding at both $\mathrm{pH}$ values when we made mutation to histidine, and the second is to prescreen $\mathrm{pH}$-dependent mutants through decreases binding at one $\mathrm{pH}$ condition ( $\mathrm{pH} 6.0$ or $\mathrm{pH} 7.4$ ). Theoretically, this method will increase the productivity of generating a $\mathrm{pH}$-dependent antibody while predicting ionizable sensitive hot-spots. Because Rojiani et al. have demonstrated that introducing single-histidine residue at the antibody $\mathrm{CDR}$ is used for $\mathrm{pH}$-dependent binding to Her2 antigen at the acidic $\mathrm{pH}$ and dissociation from it at extracellular physiological pH (Sulea et al. 2020). The second step of the stepwise approach involves single-acidic amino acid residue substitutions of the identified ionizable sensitive hot-spot residues, aiming at the second round of $\mathrm{pH}$ dependent antibody screening. Notably, compared to the wild-type antibody, only one amino acid site is mutated for the screened $\mathrm{pH}$-dependent antibodies, which can retain near original antibody structure and affinity. In this study, we divided nine different levels of $\mathrm{pH}$-dependent antibodies by the stepwise mutagenesis approach. The experimental results demonstrated the viability of the stepwise mutagenesis approach and signified the importance of an acidic amino acids for introducing $\mathrm{pH}$ dependence in antigen-antibody binding.

\section{Methods}

\section{Construction of expression vectors}

The anti-carcinoembryonic antigen (CEA) $\mathrm{mIgG} 1$ variable region was obtained using murine hybridoma cells (ATCC, Manassas, VA). A heavy-chain variable region and lightchain variable region were amplified from the cDNA of the murine hybridoma cells using primers mT84HC-VF and mT84HC-VR for the heavy chain and mT84LC-VF and mT84LC-VR for the light chain. The polymerase chain reaction (PCR) fragments of the heavy-chain and lightchain variable regions were digested using BsmbI-BswiI and BsaI-NheI (Thermo Scientific, MA, USA), respectively. The primers sequence shown in Table 1. The PCR fragments of the variable region were each introduced into the vectors LYpIgG-HC and LYp2M-LC. Constructs containing inserts with the correct orientation were selected by sequencing and naming LYpIgG1-CEAHC and LYp2M-CEALC.

\section{Quantitative enzyme-linked immunoassay}

Quantitative enzyme-linked immunoassay (Quant ELISA) was used to determine antibody expression levels in the supernatant. We coated 96-well plates with goat antihuman immunoglobin-G (IgG) (Sigma, MO, USA), and nonspecific sites were then blocked with $1 \%$ bovine serum albumin in phosphate-buffered saline (PBS; Corning, NY, USA). Dilutions (1:100) of supernatant were made in PBS and then added to the wells. Bound antibodies were detected using goat antihuman IgG antibody (Promega, WI, USA) conjugated with horseradish peroxidase (HRP). A standard curve was generated using purified human IgG isotype standard antibody (Thermo Scientific, MA, USA).

\section{Single-histidine scanning mutagenesis}

The LYpIgG1-CEAHC and LYp2M-CEALC vector sequences were used as template sequences to design mutagenesis primers for encoding the histidine residue at each position within the complementarity-determining regions (CDR; Kabat definition method) of the variable region. For the CDR residue positions, only one residue
Table 1 Primers for the amplification of the anti-CEA mIgG1 variable region

\begin{tabular}{ll}
\hline Primers & Sequence $\left(5^{\prime}-3^{\prime}\right)$ \\
\hline mT84HC-VF & CTACTACTACGTCTCAGTGTGAGGTTCAGCTGCAGCAGTC \\
mT84HC-VR & CATCATCATCGTACGTGAGGAGACGGTGACTGAGGT \\
mT84LC-VF & CTACTACTAGGTCTCAGTGTGACATTGTGCTGACCCAATCT \\
mT84LC-VR & CTACTACTAGCTAGCACGTTTTATTTCCAGCTTGGTCCCC \\
\hline
\end{tabular}

The restriction enzyme sites are written in boldface letters

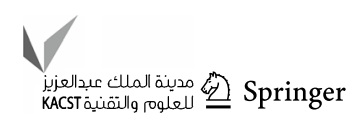


was mutated to the histidine at one time. Obtained DNA was transformed into Escherichia coli DH5 $\alpha$ cells, and suitable mutants were confirmed using DNA sequencing. The mutants were transiently transfected into Chinese hamster ovary (CHO) cells, and we performed quant ELISA measurements on the mutation antibodies to evaluate their expression quantity.

\section{Dual-pH capture ELISA}

Microtiter wells (Corning, NY, USA) were coated with $100 \mu \mathrm{L}$ of $1 \mu \mathrm{g} / \mathrm{mL}$ human CEA antigen (Abcam, MA, USA) overnight at $4{ }^{\circ} \mathrm{C}$. After being washed thrice with PBS (Corning, NY, USA), plates were blocked either with a pH 6.0 acidic buffer (Krebs-Ringer solution with $1.26 \mathrm{~g} / \mathrm{L}$ bicarbonate, $10 \mathrm{~g} / \mathrm{L} \mathrm{BSA}$, and adjust $\mathrm{pH}$ to $6.0 \mathrm{using}$ $5 \mathrm{~mol} / \mathrm{L}$ lactic acid stirring) or a $\mathrm{pH} 7.4$ slightly basic buffer (Krebs-Ringer solution with $1.26 \mathrm{~g} / \mathrm{L}$ bicarbonate, $10 \mathrm{~g} / \mathrm{L}$ $\mathrm{BSA}$ and adjust $\mathrm{pH}$ to 7.4 using $5 \mathrm{~mol} / \mathrm{L}$ lactic acid stirring). The expression supernatant of mutants and wild-type were diluted in the $\mathrm{pH} 6.0$ acidic buffer or $\mathrm{pH} 7.4$ slightly basic buffer to a final antibody concentration of $10 \mathrm{ng} / \mathrm{mL}$ and then added to the previously blocked and washed wells, followed by incubation for $1 \mathrm{~h}$ at room temperature. Diluted antihuman IgG HRP conjugate (Promega, WI, USA) using the $\mathrm{pH}$ 6.0 acidic buffer or $\mathrm{pH} 7.4$ slightly basic buffer was added to the plates, which were then incubated for $1 \mathrm{~h}$ at room temperature. The plates were then washed 3 times with the corresponding $\mathrm{pH} 6.0$ or $\mathrm{pH} 7.4$ assay buffer and removed the buffer solution from the wells as much as possible. $50 \mu \mathrm{L}$ of TMB peroxidase substrate solution (Thermo, MA, USA) was added to each well, and the reactions were stopped after 3 min with $50 \mu \mathrm{L}$ of $0.1 \mathrm{~N} \mathrm{HCl}$. The plates were read at OD $450 \mathrm{~nm}$ using a microplate spectrophotometer.

\section{lonizable sensitive hot-spot residues sequence analysis}

The National Center for Biotechnology Information (NCBI) database was used in the sequence alignment. The NCBI protein accession numbers of the anti-CEA mAb T84.66 Fv fragment were as follows: GenBank, CAA36980.1 (heavy chain), and CAA36979.1 (light chain). The six hot-spot residues were marked using Discovery Studio software (Dassault, France) with the crystal structure data of the anti-CEA mAb T84.66 Fv fragment on the Protein Data Bank (PDB) site (1J05).

\section{Generation of pH-dependent mutants}

We designed the single-residue mutation primers that would encode the aspartic acid or glutamic acid residues at six identified ionizable sensitive hot-spot residue positions to screen the $\mathrm{pH}$-dependent antibodies. The 12 mutants were transiently expressed in CHO cells to generate the antibody mutants. Quant ELISA was performed to evaluate the expression levels of the antibodies. A dual-pH capture ELISA was then performed using $10 \mathrm{ng} / \mathrm{mL}$ antibody concentration to evaluate the $\mathrm{pH}$ dependence of the mutants that bind to the CEA antigen.

\section{Antibody production and purification}

For the soluble production of $\mathrm{pH}$-dependent mutants, we transiently transfected plasmids, including wild-type and mutants, into suspension-cultured $\mathrm{CHO}$ cells separately using Polyethyleneimine Max (Sigma, MO, USA) and a serum-free medium (Thermo, MA, USA). The culture supernatant was collected 5 days after transfection. The chimeric wild-type antibody and nine $\mathrm{pH}$-dependent mutants were purified, per the manufacturer's instructions, from the culture supernatant by using the protein G Sepharose (Thermo, MA, USA). The concentration of the purified wild-type antibody and nine $\mathrm{pH}$-dependent mutants was measured on a NanoDrop $^{\mathrm{TM}} 2000$ spectrophotometer (Thermo Fisher) using absorbance at $280 \mathrm{~nm}$ and assessed the purity of antibodies using sodium dodecyl sulfate-polyacrylamide gel electrophoresis (SDS-PAGE) and Coomassie brilliant blue staining.

\section{Human CEA-binding properties of anti-CEA chimeric antibody and mutants}

To estimate the binding properties and $\mathrm{pH}$ dependence of mutants, we performed antibody concentration titration ELISA, per the procedure described in the previous section on dual-pH capture ELISA. The difference is that, in this case, the test sample, the mutants, and the wild-type antibody, were serial diluted (1:2) to 500, 250, 125, 62.5, 31.25, $15.63,7.81,3.91,1.95,0.98$, and $0.49 \mathrm{ng} / \mathrm{mL}$ in either $\mathrm{pH}$ 6.0 or $\mathrm{pH} 7.4$ buffer. The half-maximal effective concentrations (EC50) values of antibodies at $\mathrm{pH} 6.0$ and $\mathrm{pH} 7.4$ for binding activities to human CEA antigen were determined using optical density (OD) $450 \mathrm{~nm}$ value and calculated with the nonlinear fit model (variable slope, four parameters) of GraphPad Prism version 9.1.2. The $\mathrm{pH}$ dependence of antibodies was determined using Eq. 1.

$\mathrm{pH}$ dependence $=\frac{\mathrm{EC50}(\mathrm{pH} 7.4)}{\mathrm{EC50}(\mathrm{pH6} .0)}$.

Subsequently, we more fully characterized the wild-type antibody and mutants over a range of $\mathrm{pH}$ values to verify the $\mathrm{pH}$-dependent binding, and the reaction buffer of ELISA was adjusted to $\mathrm{pH} 5.5,5.8,6.0,6.2$ 6.5, 6.8, 7.0, and 7.4, with each $\mathrm{pH}$ tested in duplicate at $10 \mathrm{ng} / \mathrm{mL}$ using antigencapture ELISA. 


\section{Fluorescence-activated cell sorter (FACS) analysis of the $\mathrm{pH}$ dependence of mutant}

LS147T cells in T-75 flasks were washed twice with PBS (Corning, NY, USA). Cells were grown to $80 \%$ confluency, trypsinized, and harvested. The cells were pelleted by centrifugation and resuspended in the appropriate $\mathrm{pH}$-binding buffer, at $\mathrm{pH}$ values of either 6.0 or 7.4, and then dispensed at $1 \times 10^{6}$ cells/well in a 96-well polypropylene (PP) U-bottom plate (Merck Millipore, NJ, USA) at $4{ }^{\circ} \mathrm{C}$. Antibodies were serially diluted in $\mathrm{pH} 6.0$ assay solution ((Krebs-Ringer solution with $1.26 \mathrm{~g} / \mathrm{L}$ bicarbonate, $10 \mathrm{~g} / \mathrm{L}$ BSA, and adjust $\mathrm{pH}$ to 6.0 using $5 \mathrm{~mol} / \mathrm{L}$ lactic acid stirring) or $\mathrm{pH} 7.4$ assay solution ((Krebs-Ringer solution with $1.26 \mathrm{~g} / \mathrm{L}$ bicarbonate, $10 \mathrm{~g} / \mathrm{L} \mathrm{BSA}$, and adjust $\mathrm{pH}$ to $7.4 \mathrm{using} 5 \mathrm{~mol} / \mathrm{L}$ lactic acid stirring), then added to cells and incubated at $4{ }^{\circ} \mathrm{C}$ for $1 \mathrm{~h}$ in the dark. The cells were then washed twice by centrifugation at $1500 \mathrm{rpm}$, supernatants were removed by aspiration, and the cells were resuspended in $200 \mu \mathrm{L}$ binding buffer at $4{ }^{\circ} \mathrm{C}$. Detection reagent, fluorescein (FITC)-conjugated affinipure goat antihuman IgG FITC (Proteintech, IL, USA), was then added, and samples were incubated at $4{ }^{\circ} \mathrm{C}$ for $45 \mathrm{~min}$ in the dark. The cells were washed twice in $200 \mu \mathrm{L}$ of pHbinding buffer before being analyzed by BD FACSCalibur flow cytometry (BD, NJ, USA). The half-maximal effective concentrations (EC50) values of antibodies at pH6.0 and pH7.4 for binding activities to human CEA antigen were determined using Freq. of Parent (\%) and calculated with the nonlinear fit model (variable slope, four parameters) of GraphPad Prism version 9.1.2. The $\mathrm{pH}$ dependence of antibodies was determined using Eq. 1.

\section{Results}

\section{Single-residue histidine scanning mutagenesis}

The mutagenesis approach of $\mathrm{pH}$-dependent antibodies was designed as shown in Fig. 1. To determine the position of ionizable sensitive hot-spot residues suitable for acidic amino acid insertion sites, we performed single-residue histidine scanning mutagenesis to evaluate the binding of mutants with CEA antigen using dual-pH capture ELISA at $10 \mathrm{ng} / \mathrm{mL}$. As displayed in Fig. 2a, b, the binding affinities of the six mutants were significantly lower than that of the wild-type chimera antibody. Therefore, we inferred that the ionizable sensitive hot-spot residues were the HC-P98, HC-G100, and HC-A107 residues located at the heavy-chain CDR3 region and the LC-F036, LC-R54, and LC-T95 residues located at the light-chain CDRs, and the six residues should be suitable sites for acidic amino acid substitution to screen $\mathrm{pH}$-dependent antibodies.

\section{Ionizable sensitive hot-spot residues sequence analysis}

For a further analysis of the identified Ionizable sensitive hot-spot residues sequence, the structural localization of LC-F36, LC-R54, LC-T95, HC-P98, HC-G100, and HC-A107 in the anti-CEA T84.66 Fv region was investigated using the crystal structure data of the anti-CEA $\mathrm{mAb}$ T84.66 Fv fragment on the PDB site (1J05). As shown in Fig. 2c, the six hot-spot residues were notably close to each other and were surface residues of the antibody CDR.

\section{Generation of pH-dependent mutants}

Subsequently, we performed single-residue acidic amino acid (aspartic acid or glutamic acid) mutagenesis at the six ionizable sensitive hot-spot residues to evaluate the $\mathrm{pH}$ dependence through a dual-pH capture ELISA assay. As illustrated in Fig. 3 and Table 2, the binding of the mutants of LF36D, LF36E, LR54D, LR54E, LT95D, HP98D, HG100D, HA107D, and HA107E was dependent on $\mathrm{pH}$. Their binding affinity ratios value ranged from 2.09 to 14.94 at the $\mathrm{pH}$ values of 6.0 and 7.4. However, the binding response signal of all mutants was low than wild-type antibodies. Furthermore, the HP98D, HG100D, HA107E, and HA107D mutants exhibited even lower binding to the CEA antigen, which decreased the binding response signal by more than $50 \%$ compared to wild-type at a $\mathrm{pH}$ of 6.0 .

\section{Antibody production and purification}

For a further analysis of the $\mathrm{pH}$ dependence of the $\mathrm{pH}$ dependent mutants, the use of high-purity $\operatorname{IgG}$ is necessary. To determine the purity and molecular weight of the purified $\mathrm{IgG}$, we performed SDS-PAGE and Coomassie staining. As shown in Fig. 4, two evident bands of IgG, the heavy chains at approximately $55 \mathrm{kDa}$ and light chains at approximately $25 \mathrm{kDa}$, were present in the stained gel, indicating that the purity of all IgGs satisfied the requirements for all subsequent experiments.

\section{Human CEA-binding properties of anti-CEA chimeric antibody and mutants}

To investigate the binding properties of the nine mutants, we performed the antibody concentration titration capture ELISA at the $\mathrm{pH}$ values of 6.0 and 7.4. As detailed in Fig. 5a, b, all mutants were bound to the CEA in a pH-dependent manner. However, the binding of all mutants was low than wild-type antibodies. The binding activity of the optimal performing LF36E and LF36D mutants were intensely weaker at the neutral $\mathrm{pH}$ of 7.4 than at the weakly acidic $\mathrm{pH}$ of 6.0. The resulting EC50 value for $\mathrm{pH} 6.0$ to $\mathrm{pH} 7.4$ of the LF36E and LF36D 


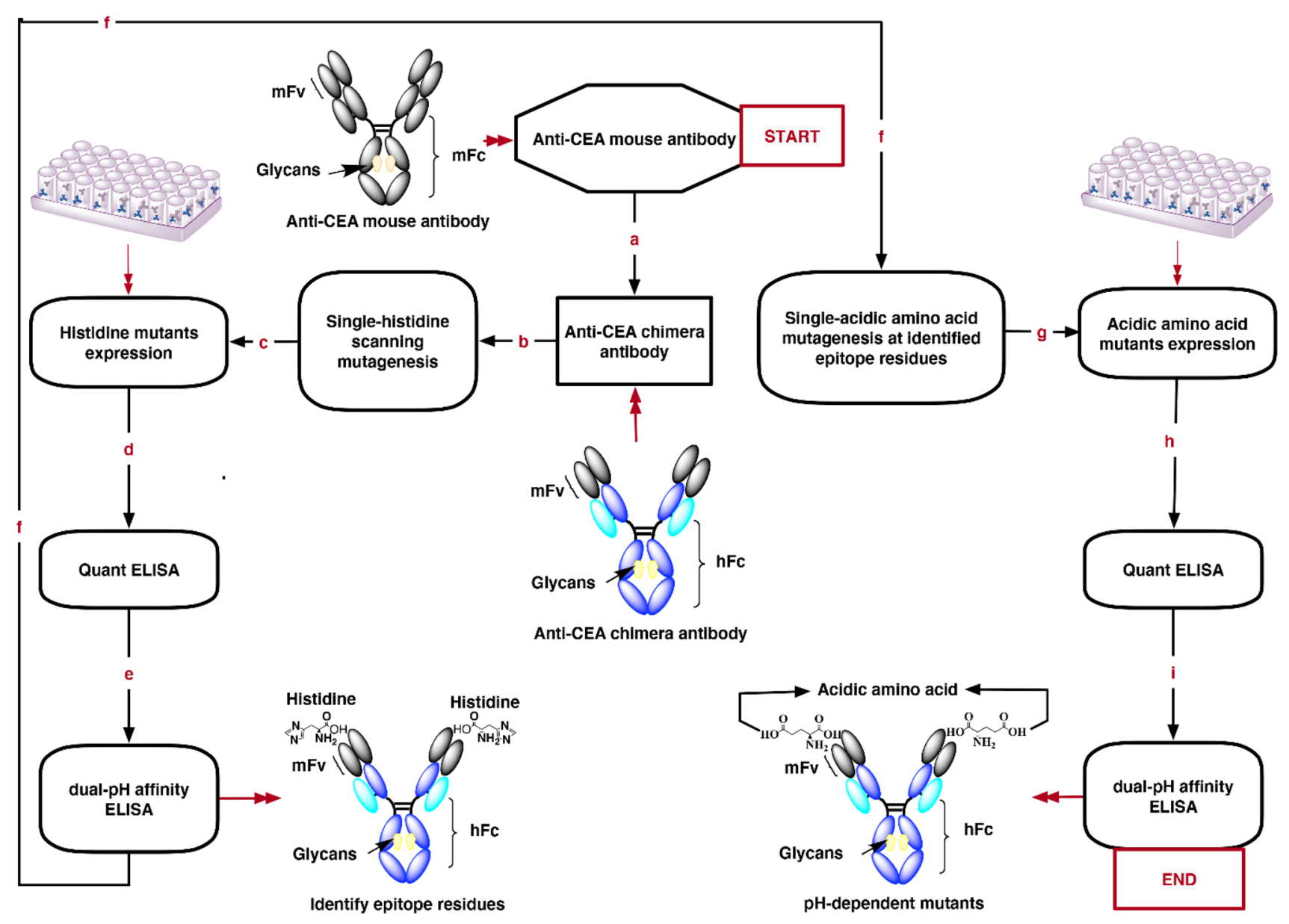

Fig. 1 Schematic diagram of mutagenesis strategy for the generation of $\mathrm{pH}$-dependent mutants. a Construction of the anti-CEA chimera antibody. b Single-histidine scanning mutagenesis. c Expression of histidine mutants. d Measurement of the expression quantity of histidine mutants. e Determination of epitope residues using dual-pH

mutants were 32.86 and 29.36. Furthermore, the other seven mutants also exhibited different levels of $\mathrm{pH}$ dependence, but which were lower than that of the LF36E and LF36D mutants (Table 3). However, the $\mathrm{pH}$ dependence of the wild-type antibody was not observed in the dual-pH capture ELISA experiments. When the purified mutants were characterized at a broad $\mathrm{pH}$ range from $\mathrm{pH} 5.5$ to $\mathrm{pH} 7.4$, as shown in Fig. 5c, the binding signal revealed that the level monotonically decreased with increased $\mathrm{pH}$ levels. However, the binding of the wildtype antibody barely decreased with $\mathrm{pH}$. The purified LF36E and LF36D mutants exhibited extreme $\mathrm{pH}$ sensibility and higher affinity than other mutants at the $\mathrm{pH}$ range of 5.5-6.0.

\section{Flow cytometry analysis of LF36D and LF36E mutant in cellular CEA binding}

Hallmarks of the TME are a lower extracellular $\mathrm{pH}$ due to the increased lactic acid secretion resulting from capture ELISA. f Single-residue acidic amino acid mutagenesis at identified epitope residue. $\mathbf{g}$ Expression of acidic amino acid mutants. h Measurement of the expression quantity of acidic amino acid mutants. i Screening of $\mathrm{pH}$-dependent mutants using dual-pH capture ELISA

glycolytic tumor metabolism even under aerobic conditions [i.e., the Warburg effect (Warburg 1925, 1956)]. LF36E and LF36D were two of the most $\mathrm{pH}$-sensitive (based on ELISA). The pH selectivity of LF36D and LF36E was also tested by flow cytometry using LS147T cells expressing human CEA on the cell surface. These two mutants slightly reduced binding to LS147T cells at pH6.0 compared to wild-type, while all had substantially reduced binding at pH7.4 (Fig. 6a). However, as shown in Fig. 6b, c, the calculated effective concentration, 50\% (EC50) values for wild-type antibody shows undesirable stronger binding at $\mathrm{pH} 7.4(22.80 \mathrm{ng} / \mathrm{mL})$ relative to pH6.0, while the binding of clones LF36D and LF36E decreases to only 12 to $10 \%$ at $\mathrm{pH} 7.4$ relative to $\mathrm{pH} 6.0$ (i.e., a 8- to tenfold change as estimated based on the data shown in Fig. 6c). 
a Single-histidine scanning mutagenesis of heavy chain CDRs region
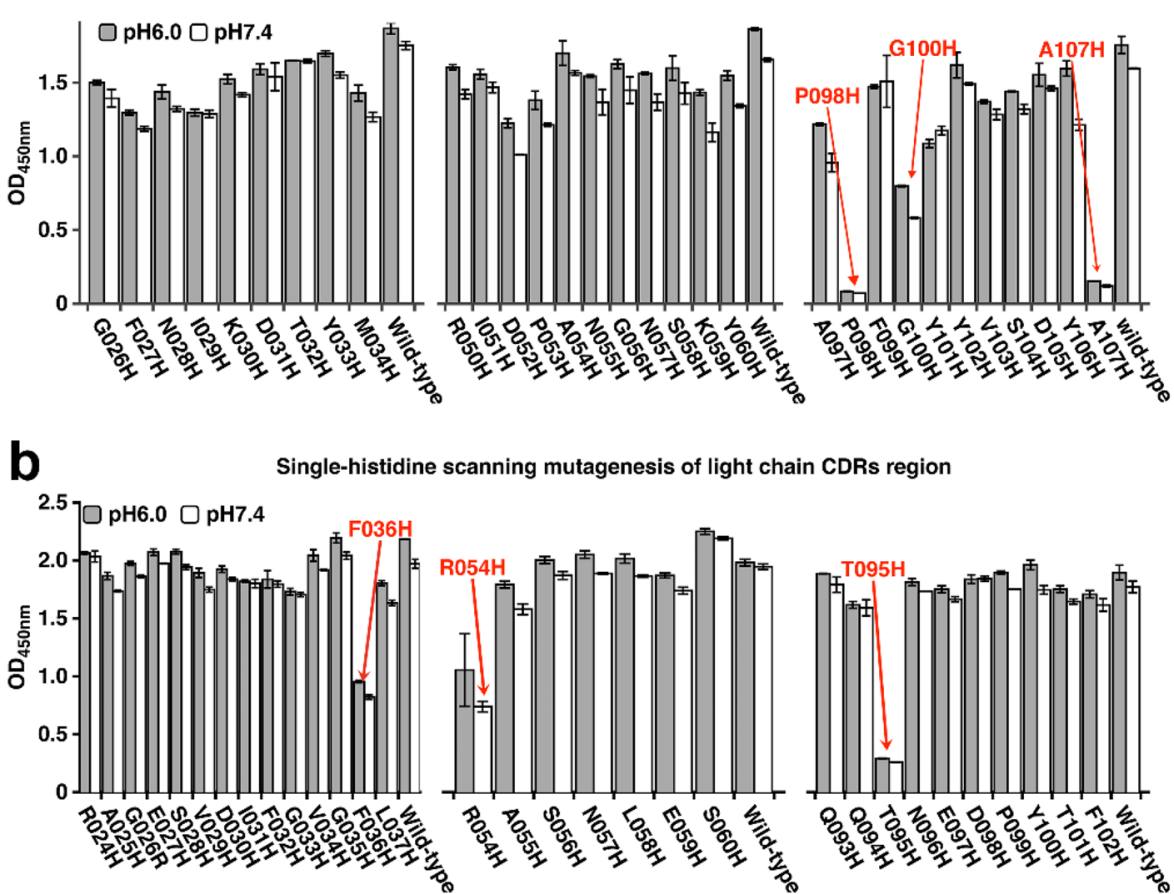

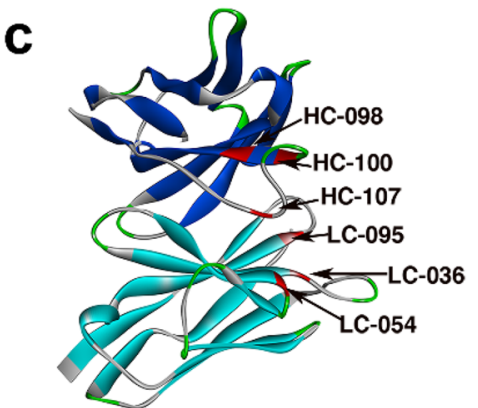

Anti-CEA antibody T84.66 Fv fragment
Fig. 2 Screening of pH-dependent mutants. a, b Determination of the epitope residues for the CDR domain of the anti-CEA antibody using dual-pH capture ELISA. Error bars represent standard deviations between technical replicates. $\mathbf{c}$ Analysis of relevant anti-CEA antibody epitope residues. A ribbon diagram of the crystal structure of an anti-CEA T84.66 Fv fragment is presented. The heavy chain is in dark blue, and the light chain is in light blue. The IgG ionizable sensitive hot-spot residues (LC-F36, LC-R54, LC-T95, HC-P98, HC-G100, and HC-A107) are represented as red ribbons
Single-acidic animo acid mutagenesis screening of identified epitope residues

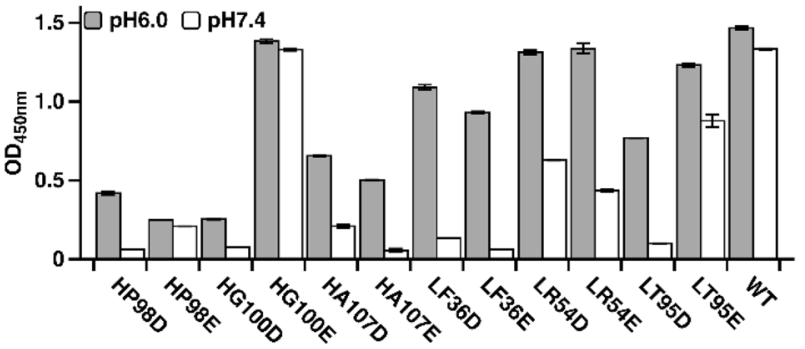

Fig. 3 Screening of the $\mathrm{pH}$-dependent mutants using dual-pH capture ELISA. Error bars represent standard deviations between technical replicates

\section{Discussion}

We successfully isolated $\mathrm{pH}$-dependent antibodies that targeted the acidic $\mathrm{pH}$ environment using a stepwise approach based on single-reside histidine scanning mutagenesis and acidic amino acid mutagenesis, as shown in Fig. 1. In this study, pH-dependent antibodies were not obtained through histidine scanning mutagenesis in the first round
Table 2 Dual-pH ELISA data of acidic amino acid mutants

\begin{tabular}{lllll}
\hline Antibody & \multicolumn{2}{l}{$\mathrm{OD}_{450}$ value } & $\mathrm{OD}_{450}$ percentage & $\mathrm{OD}_{450}$ Ratio \\
\cline { 2 - 3 } & $\mathrm{pH6.0}$ & $\mathrm{pH} 7.4$ & $\mathrm{pH6.0/WT}$ & $\mathrm{pH6.0/pH7.4}$ \\
\hline Wild-type & 1.467 & 1.334 & $100.0 \%$ & 1.10 \\
LF36D & 1.091 & 0.134 & $74.4 \%$ & 8.14 \\
LF36E & 0.926 & 0.062 & $63.1 \%$ & 14.94 \\
LR54D & 1.312 & 0.629 & $89.4 \%$ & 2.09 \\
LR54E & 1.336 & 0.436 & $91.1 \%$ & 3.06 \\
LT95D & 0.770 & 0.101 & $52.5 \%$ & 7.62 \\
LT95E & 1.232 & 0.878 & $84.0 \%$ & 1.40 \\
HP98D & 0.419 & 0.063 & $28.6 \%$ & 6.65 \\
HP98E & 0.250 & 0.210 & $17.0 \%$ & 1.19 \\
HG100D & 0.255 & 0.077 & $17.4 \%$ & 3.31 \\
HG100E & 1.383 & 1.330 & $94.3 \%$ & 1.04 \\
HA107D & 0.656 & 0.210 & $44.7 \%$ & 3.12 \\
HA107E & 0.503 & 0.057 & $34.3 \%$ & 8.82 \\
\hline
\end{tabular}

Association ELISA-OD450 values of acidic amino acid mutants and wild-type antibody at $\mathrm{pH} 6.0$ and $\mathrm{pH} 7.4$, their percentage to an OD450 value of wild-type antibody at $\mathrm{pH} 6.0$, and their $\mathrm{pH} 6.0$ to $\mathrm{pH}$ 7.4 OD450 value ratio are shown. The OD450 values represent the average of duplicates 


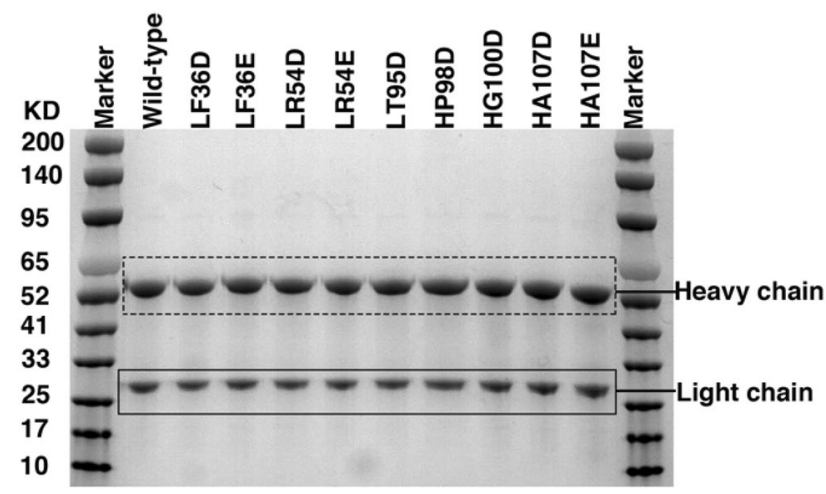

Fig. 4 SDS-PAGE of purified antibody. The monomeric anti-CEA antibody heavy chain is indicated by $55 \mathrm{KD}$, and the light chain is indicated by $25 \mathrm{KD}$. Lane 1,12: size markers (kDa). Lane 2: wild-type antibodies. Lane 3-11: pH-dependent mutants

of screening (Fig. 2a, b), but these results were unsurprising. Single-histidine residues can introduce the same $\mathrm{pH}$ dependence as a single aspartic acid or glutamic acid residue. This is because the $\mathrm{pH}$-dependence effect is governed by the underlying thermodynamics, namely, the $\mathrm{pKa}$ changes of the ionizable group regardless of whether the group is histidine or aspartic/glutamic acid. The $\mathrm{pH}$ dependence effect is also governed by the unique structural/chemical details of each unique antibody-antigen interaction. The introduction of multiple ionizable groups undergoing $\mathrm{pKa}$ changes has the highest potential for achieving potent $\mathrm{pH}$ dependence (Murtaugh et al. 2011).

In this study, we analyzed the spatial sequence characteristics of the hot-spot identified by histidine scanning (Fig. 2c). Murtaugh et al. used computer-aided sequence analysis for $\mathrm{pH}$-dependent antibody engineering (Murtaugh et al. 2011), and found that residues within the interface are poor histidine hosts, while peripheral residues are well suited for histidine substitution. In the current study, the six identified ionizable sensitive hot-spot were prominently located on the surface of the antibody structure. The results demonstrated that single-residue histidine mutagenesis scanning could be applied similar to computer-aided sequence analysis to help identify $\mathrm{pH}$-sensitive clones.

In the second step, the screening yielded nine $\mathrm{pH}$-dependent antibodies by introducing acidic amino acid mutations at the ionizable sensitive hot-spot residue sites (Figs. 3 and $5)$. This result is similar to several previous reports that introduced $\mathrm{pH}$ dependence into the protein-protein interface via acidic amino acid substitution. For example, Stennicke et al. reported that the $\mathrm{pH}$-dependent binding of carboxypeptidase $\mathrm{Y}$ and substrate was achieved by inserting aspartic acid and glutamic acid residues into the active site of carboxypeptidase Y. Wallace and Shen discovered that introducing glutamate residues into the active sites at low $\mathrm{pH}$ values could induce the formation of silk protein dimers. They ascribed this to the deprotonation of glutamic acid and aspartic acid, both of which are involved in protein substrate binding (Stennicke et al. 1994; Wallace and Shen 2012). Therefore, in this study, we concluded that deprotonation of acidic amino acid side chains affected the electrostatic interaction between the antibody and the CEA target, driving the pH-dependent interaction (Gera et al. 2012; Sagermann et al. 2009). In addition, according to the theory of proteinassociated chemical switches (PaCS) reported by Chang H W Frey G et al. using physiological chemicals greatly expands the success rate and degree of selectivity of $\mathrm{pH}-$ selective antibodies compared to solely histidine-dependent protein selectivity. We used physiological chemicals in the buffer, such as sodium bicarbonate, which can also facilitate the introduction of $\mathrm{pH}$ dependence.

However, a phenomenon was observed in Figs. 3, 5, and 6, which is the binding response signal of all mutants, decreased compared to wild-type at a $\mathrm{pH}$ of 6.0. The phenomenon verified that the initial structure of the antibody was altered by introducing the acidic amino acid. This is because the expected $\mathrm{pKa}$ value shifts for these acidic amino acids into the physiological range would require a significant cost in free energy, resulting in protein destabilization (Isom et al. 2008, 2011). Nevertheless, we can still improve the binding affinity of the $\mathrm{pH}$-dependent mutants using antibody-antigen affinity maturation.

The pH selectivity of clones LF36E and LF36D was tested by flow cytometry using LS147T cells expressing human CEA on the cell surface (Fig. 6a). LF36E and LF36D were two of the most $\mathrm{pH}$-sensitive (based on ELISA) and displayed 8- and tenfold higher $\mathrm{pH}$ sensitivity, respectively. Compared to the ELISA results, the flow cytometry results indicated a more modest $\mathrm{pH}$ dependence. We speculated LS147T cells under normal physiological conditions (pH7.4) possess better cellular vitality, and therefore, cells could achieve much cellar uptake of salt ions compared with cells under the acidic condition (pH6.0). The decreased salt ions in physiological $\mathrm{pH}$ conditions $(\mathrm{pH} 7.4)$ could have dampened the selectivity in the flow cytometry assay. Chang H W, Frey G, et al. have proved that the decrease of salt concentration (e.g., sodium bicarbonate and sodium chloride) can increase antigen-antibody binding. These authors found a sixfold loss in pH selectivity in the absence of sodium bicarbonate and the presence of sodium chloride (Chang et al. 2021). 
a

Antibody-concentration titration ELISA

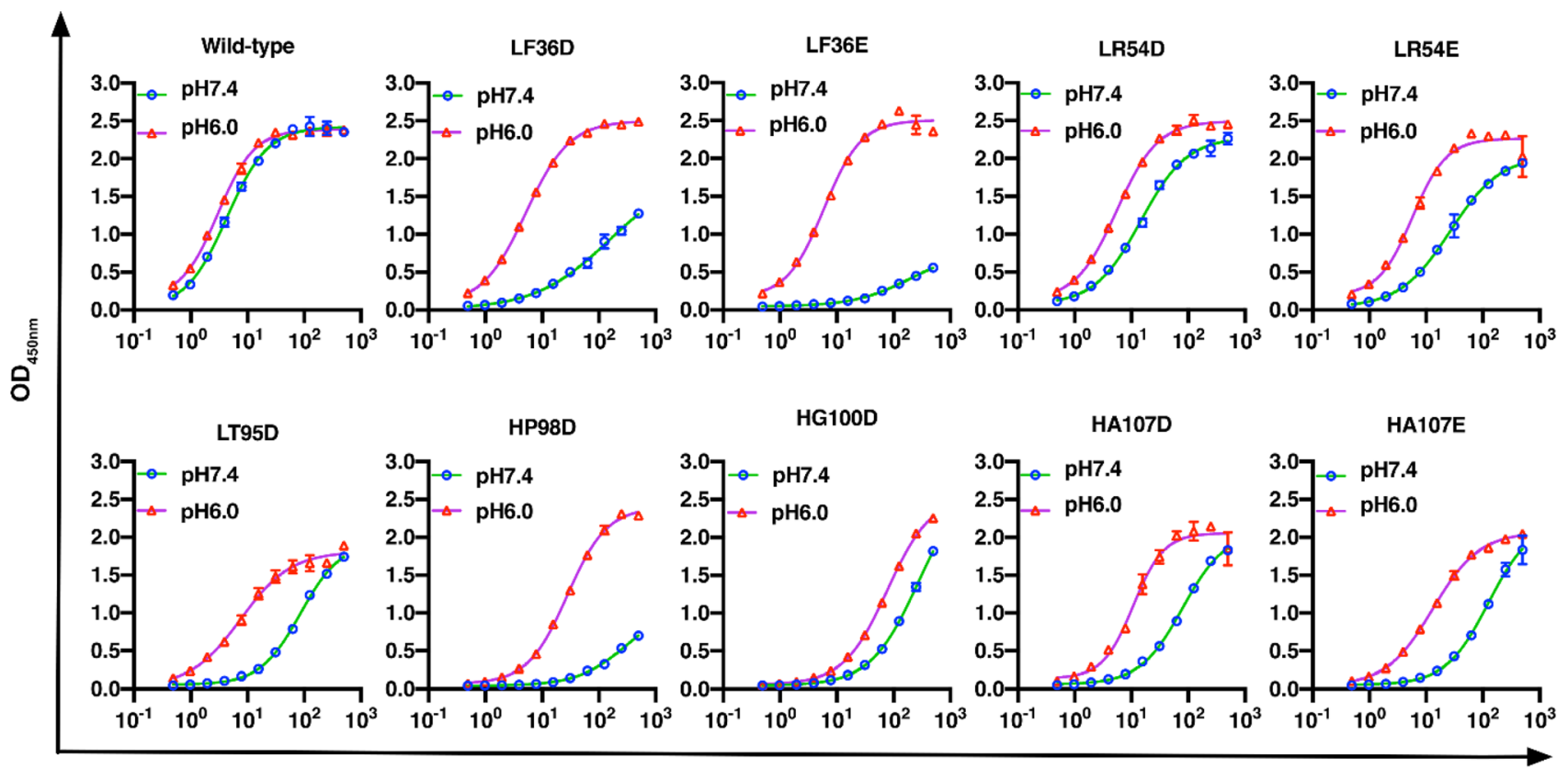

Antibody concentration $(\mathrm{ng} / \mathrm{mL})$

b

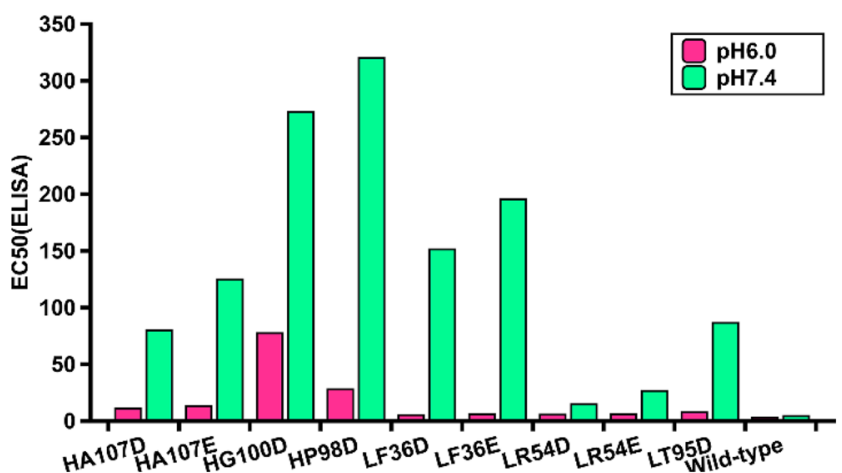

Fig. 5 Characterization of purified $\mathrm{pH}$-dependent mutants binding to CEA. a The $\mathrm{pH}$ dependence of anti-CEA antibody mutants binding to CEA antigen was tested under acidic and physiological $\mathrm{pH}$ conditions, and CEA binding was analyzed through a titration ELISA. b EC50 values of anti-CEA mutants binding to human CEA by ELISA at pH6.0 and $\mathrm{pH} 7.4$ were calculated using the nonlinear fit (variable
C

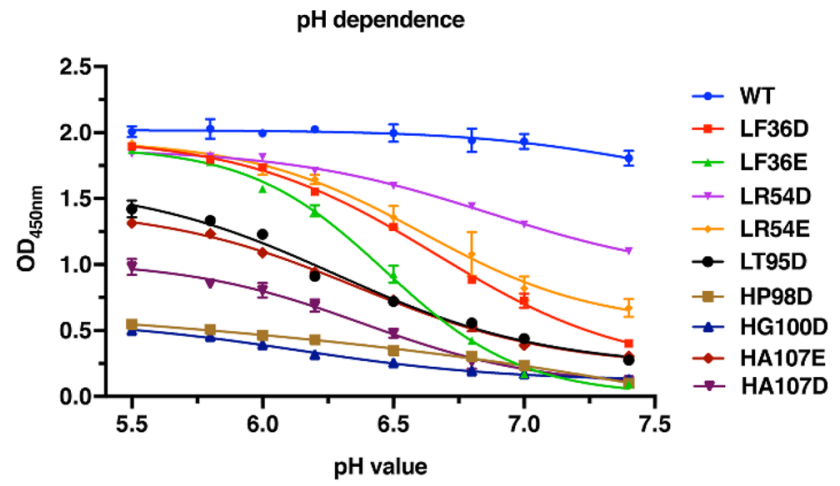

slope, four parameters) model built into GraphPad Prism software version 9.1.2. $\mathbf{c}$ The binding affinity of tested CEA antibody mutants from binding experiments to CEA antibody at a broad $\mathrm{pH}$ value using a $\mathrm{pH}$ range ELISA. Error bars represent standard deviations between technical replicates 
Table 3 EC50 value of purified pH-dependent mutants and wild-type binding to recombinant human CEA

\begin{tabular}{|c|c|c|c|}
\hline \multirow[t]{2}{*}{ Antibody } & \multicolumn{3}{|c|}{ EC50 (ng/ml) } \\
\hline & $\mathrm{pH} 7.4$ & pH6.0 & $\mathrm{pH} 7.4 / \mathrm{pH} 6.0$ \\
\hline Wild-type & 4.328 & 2.996 & 1.45 \\
\hline LF36D & 151.300 & 5.154 & 29.36 \\
\hline LF36E & 195.200 & 5.940 & 32.86 \\
\hline LR54D & 14.630 & 5.498 & 2.66 \\
\hline LR54E & 26.440 & 5.744 & 4.60 \\
\hline LT95D & 86.450 & 7.623 & 11.34 \\
\hline HP98D & 320.000 & 27.770 & 11.52 \\
\hline HG100D & 272.200 & 77.400 & 3.52 \\
\hline HA107D & 79.990 & 10.760 & 7.43 \\
\hline HA107E & 124.800 & 13.150 & 9.49 \\
\hline
\end{tabular}

Absolute EC50 values were derived from the nonlinear least squares fit of at two measurements of the ELISA-OD450 value. Binding affinity (EC50) of mutants and wild-type antibody at $\mathrm{pH} 7.4$ and $\mathrm{pH} 6.0$, their $\mathrm{pH} 7.4$ to $\mathrm{pH} 6.0$ binding affinity (EC50) ratio are shown as $\mathrm{pH}$ dependence

\section{Conclusions}

In summary, this study provides a new method for engineering $\mathrm{pH}$-dependent antibodies toward acidic microenvironments. In addition, we found that single-residue histidine scanning mutagenesis was ideal for identifying the location of inserted ionizable residues (e.g., aspartic acid and glutamic acid) to introduce $\mathrm{pH}$ dependence into the protein-protein reaction interface. This study provides new ideas for hot-spot analysis for drug discovery applications that target protein-protein interactions. In addition, this study provides a new approach for engineering $\mathrm{pH}$-dependent antibodies toward acidic microenvironments. Our results also support the use of acidic amino acid mutagenesis for $\mathrm{pH}$-selective engineering in other medical applications, such as those involving $\mathrm{pH}$-dependent antimicrobial peptides and antimicrobial proteins (Kacprzyk et al. 2007). In particular, single-residue mutagenesis is suitable for screening $\mathrm{pH}$ dependent binding of short amino acid sequences, such as cell-penetrating peptides, that selectively interact with the surface of tumor cells. In addition, more than two identified

a
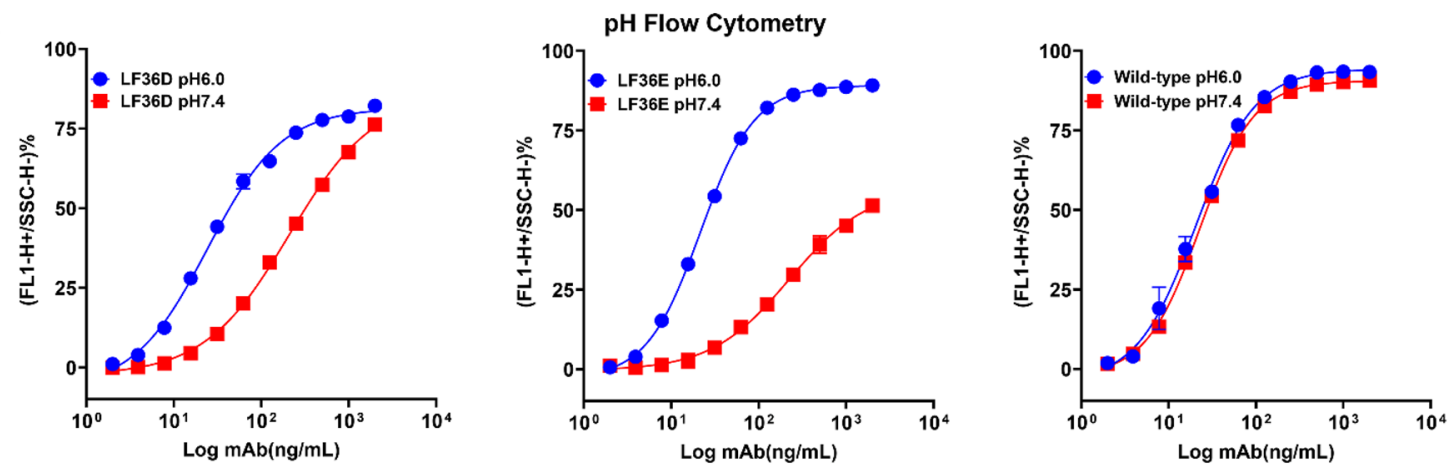

b

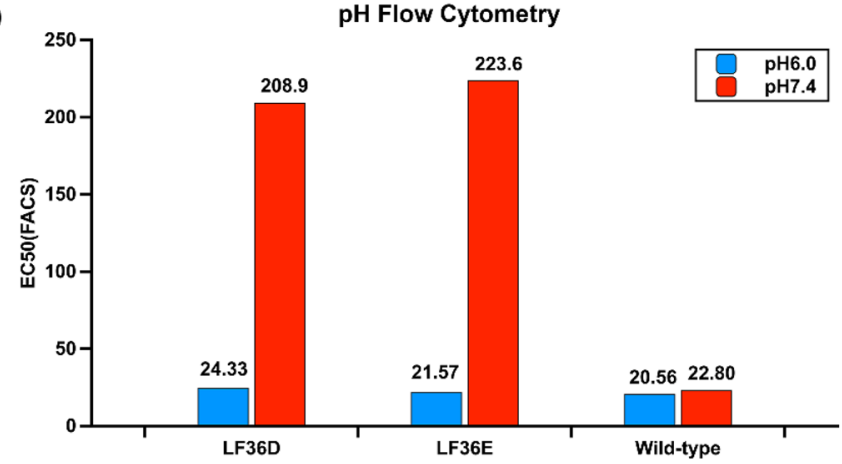

C

\begin{tabular}{cccc}
\multicolumn{4}{c}{ EC50 Values determined by pH Flow Cytometry } \\
\hline Antibody & $\begin{array}{c}\text { EC50 (ng/mL) } \\
\mathrm{pH} \mathrm{6.0}\end{array}$ & $\begin{array}{c}\mathrm{EC50}(\mathrm{ng} / \mathrm{mL}) \\
\mathrm{pH} \mathrm{7.4}\end{array}$ & $\begin{array}{c}\text { Fold change } \\
(\mathrm{pH6} 6 / 0 / \mathrm{pH7} \text { 7) }\end{array}$ \\
Wild-type & 20.56 & 22.80 & 1.11 \\
LF36D & 24.33 & 208.9 & 8.59 \\
LF36E & 21.57 & 223.6 & 10.37 \\
\hline
\end{tabular}

Fig. 6 The pH selectivity of anti-CEA mutants was determined by FACS. a Binding activity of clone LF36D and LF36E to LS147T cells at $\mathrm{pH} 6.0$ and $\mathrm{pH}$ 7.4. Error bars represent standard deviations between technical replicates. b EC50 values of anti-CEA mutants binding to human-CEA by FACS at $\mathrm{pH} 6.0$ and $\mathrm{pH} 7.4$ were calculated using the nonlinear fit (variable slope, four parameters) model built into GraphPad Prism software version 9.1.2. c The fold-change of EC50 Values was determined by $\mathrm{pH}$ Flow Cytometry at $\mathrm{pH} 6.0$ and $\mathrm{pH} 7.4$ 
hot-spot residues could be simultaneously substituted by acidic amino acids to achieve highly $\mathrm{pH}$-dependent mutants.

Acknowledgements This study was supported from the project of "Dengfeng Plan" from Foshan Hospital of Traditional Chinese Medicine (202000205) and the Science and Technology Department of Sichuan Province, China (2017SYXHZ0039).

Author contributions All authors contributed to the study's conception and design. Material preparation, data collection, and analysis were performed by WZ, CH, QS, KZ, HG, RS, and YL. The first draft of the manuscript was written by WZ, and all authors commented on previous versions of the manuscript. All authors read and approved the final manuscript.

\section{Declarations}

Conflict of interest The authors declare no conflict of interest.

\section{References}

Anderson M, Moshnikova A, Engelman DM, Reshetnyak YK, Andreev OA (2016) Probe for the measurement of cell surface $\mathrm{pH}$ in vivo and ex vivo. Proc Natl Acad Sci 113:8177-8181. https://doi.org/ $10.1073 /$ pnas. 1608247113

Ariffin AB, Forde PF, Jahangeer S, Soden DM, Hinchion J (2014) Releasing pressure in tumors: what do we know so far and where do we go from here? A review. Cancer Res 74:2655-2662. https:// doi.org/10.1158/0008-5472.CAN-13-3696

Bazban-Shotorbani S, Hasani-Sadrabadi MM, Karkhaneh A, Serpooshan V, Jacob KI, Moshaverinia A, Mahmoudi M (2017) Revisiting structure-property relationship of $\mathrm{pH}$-responsive polymers for drug delivery applications. J Control Release 253:46-63. https://doi.org/10.1016/j.jconrel.2017.02.021

Bonvin P, Venet S, Fontaine G, Ravn U, Gueneau F, Kosco-Vilbois M, Proudfoot AE, Fischer N (2015) De novo isolation of antibodies with $\mathrm{pH}$-dependent binding properties. Paper presented at: MAbs (Taylor \& Francis). https://doi.org/10.1080/19420862. 2015.1006993

Chang HW, Frey G, Liu H, Xing C, Steinman L, Boyle WJ, Short JM (2021) Generating tumor-selective conditionally active biologic anti-CTLA4 antibodies via protein-associated chemical switches. Proc Natl Acad Sci U S A. https://doi.org/10.1073/ pnas. 2020606118

Cruz-Monserrate Z, Roland CL, Deng D, Arumugam T, Moshnikova A, Andreev OA, Reshetnyak YK, Logsdon CD (2014) Targeting pancreatic ductal adenocarcinoma acidic microenvironment. Sci Rep 4:1-8. https://doi.org/10.1038/srep04410

Cunningham BC, Wells JA (1989) High-resolution epitope mapping of hGH-receptor interactions by alanine-scanning mutagenesis. Science 244:1081-1085. https://doi.org/10.1126/science.24712 67

Cunningham BC, Henner DJ, Wells JA (1990) Engineering human prolactin to bind to the human growth hormone receptor. Science 247:1461-1465. https://doi.org/10.1126/science.2321008

Gera N, Hill AB, White DP, Carbonell RG, Rao BM (2012) Design of $\mathrm{pH}$ sensitive binding proteins from the hyperthermophilic Sso7d scaffold. PLoS ONE 7:e48928. https://doi.org/10.1371/journal. pone. 0048928

Gerweck LE, Seetharaman K (1996) Cellular pH gradient in tumor versus normal tissue: potential exploitation for the treatment of cancer. Can Res 56:1194-1198. https://doi.org/10.1002/(SICI) 1097-0142(19960315)77:6\%3c1214::AIDCNCR31\%3e3.0.CO;2-

Haraya K, Tachibana T, Igawa T (2019) Improvement of pharmacokinetic properties of therapeutic antibodies by antibody engineering. Drug Metab Pharmacokinet 34:25-41. https://doi.org/10.1016/j. dmpk.2018.10.003

Hong S-T, Su Y-C, Wang Y-J, Cheng T-L, Wang Y-T (2021) Anti-tnf alpha antibody humira with ph-dependent binding characteristics: a constant-ph molecular dynamics, gaussian accelerated molecular dynamics, and in vitro study. Biomolecules 11:334. https://doi. org/10.3390/biom 11020334

Igawa T, Mimoto F, Hattori K (2014) pH-dependent antigen-binding antibodies as a novel therapeutic modality. Biochim Biophys Acta (BBA)-Proteins Proteomics 1844:1943-1950. https://doi.org/10. 1016/j.bbapap.2014.08.003

Isom DG, Cannon BR, Castañeda CA, Robinson A (2008) High tolerance for ionizable residues in the hydrophobic interior of proteins. Proc Natl Acad Sci 105:17784-17788

Isom DG, Castañeda CA, Cannon BR (2011) Large shifts in pKa values of lysine residues buried inside a protein. Proc Natl Acad Sci 108:5260-5265. https://doi.org/10.1073/pnas.0805113105

Kacprzyk L, Rydengård V, Mörgelin M, Davoudi M, Pasupuleti M, Malmsten M, Schmidtchen A (2007) Antimicrobial activity of histidine-rich peptides is dependent on acidic conditions. Biochim Biophys Acta (BBA)-Biomembr 1768:2667-2680. https://doi.org/ 10.1016/j.bbamem.2007.06.020

Kroemer G, Pouyssegur J (2008) Tumor cell metabolism: cancer's Achilles' heel. Cancer Cell 13:472-482. https://doi.org/10.1016/j. ccr.2008.05.005

Kumar DP, Tiwari A, Bhat R (2004) Effect of pH on the stability and structure of yeast hexokinase A: acidic amino acid residues in the cleft region are critical for the opening and the closing of the structure. J Biol Chem 279:32093-32099. https://doi.org/10.1074/ jbc.M313449200

Liu S, Liu C, Deng L (2018) Machine learning approaches for proteinprotein interaction hot spot prediction: progress and comparative assessment. Molecules. https://doi.org/10.3390/molecules231025 35

Murtaugh ML, Fanning SW, Sharma TM, Terry AM, Horn JR (2011) A combinatorial histidine scanning library approach to engineer highly pH-dependent protein switches. Protein Sci 20:1619-1631. https://doi.org/10.1002/pro.696

Roche S, Bressanelli S, Rey FA, Gaudin Y (2006) Crystal structure of the low-pH form of the vesicular stomatitis virus glycoprotein G. Science 313:187-191. https://doi.org/10.1126/science.1127683

Rohani N, Hao L, Alexis MS, Joughin BA, Krismer K, Moufarrej MN, Soltis AR, Lauffenburger DA, Yaffe MB, Burge CB (2019) Acidification of tumor at stromal boundaries drives transcriptome alterations associated with aggressive phenotypes. Cancer Res 79:1952-1966. https://doi.org/10.1158/0008-5472.CAN-18-1604

Rötzschke O, Lau JM, Hofstätter M, Falk K, Strominger JL (2002) A $\mathrm{pH}$-sensitive histidine residue as control element for ligand release from HLA-DR molecules. Proc Natl Acad Sci U S A 99:1694616950. https://doi.org/10.1073/pnas.212643999

Sagermann M, Chapleau RR, DeLorimier E, Lei M (2009) Using affinity chromatography to engineer and characterize $\mathrm{pH}$-dependent protein switches. Protein Sci 18:217-228. https://doi.org/10.1002/ pro. 23

Sarkar CA, Lowenhaupt K, Horan T, Boone TC, Tidor B, Lauffenburger DA (2002) Rational cytokine design for increased lifetime and enhanced potency using $\mathrm{pH}$-activated "histidine switching." Nat Biotechnol 20:908-913. https://doi.org/10.1038/nbt725

Slaga D, Ellerman D, Lombana TN, Vij R, Li J, Hristopoulos M, Clark R, Johnston J, Shelton A, Mai E (2018) Avidity-based binding to HER2 results in selective killing of HER2-overexpressing cells

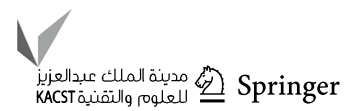


by anti-HER2/CD3. Sci Transl Med. https://doi.org/10.1126/scitr anslmed.aat5775

Stennicke HR, Mortensen UH, Christensen U, Remington SJ, Breddam K (1994) Effects of introduced aspartic and glutamic acid residues on the Pi substrate specificity, $\mathrm{pH}$ dependence and stability of carboxypeptidase Y. Protein Eng Des Sel 7:911-916. https://doi. org/10.1093/protein/7.7.911

Strauch EM, Fleishman SJ, Baker D (2014) Computational design of a pH-sensitive IgG binding protein. Proc Natl Acad Sci U S A 111:675-680. https://doi.org/10.1073/pnas.1313605111

Sulea T, Rohani N, Baardsnes J, Corbeil CR., Deprez C, CeperoDonates Y, Robert A, Schrag JD, Parat M, Duchesne M (2020). Structure-based engineering of $\mathrm{pH}$-dependent antibody binding for selective targeting of solid-tumor microenvironment. Paper presented at: MAbs (Taylor \& Francis). https://doi.org/10.1002/ 9780470669891.ch4

Szot C, Saha S, Zhang XM, Zhu Z, Hilton MB, Morris K, Seaman S, Dunleavey JM, Hsu K-S, Yu G-J (2018) Tumor stroma-targeted antibody-drug conjugate triggers localized anticancer drug release. J Clin Investig 128:2927-2943. https://doi.org/10.1172/ JCI120481

Tang H, Zhao W, Yu J, Li Y, Zhao C (2019) Recent development of $\mathrm{pH}$-responsive polymers for cancer nanomedicine. Molecules 24:4. https://doi.org/10.3390/molecules24010004
Traxlmayr MW, Lobner E, Hasenhindl C, Stadlmayr G, Oostenbrink C, Rüker F, Obinger C (2014) Construction of pH-sensitive Her2binding IgG1-Fc by directed evolution. Biotechnol J 9:1013-1022. https://doi.org/10.1002/biot.201300483

Vaupel P (2010) Metabolic microenvironment of tumor cells: a key factor in malignant progression. Exp Oncol 32:125-127

Wallace JA, Shen JK (2012) Unraveling a trap-and-trigger mechanism in the $\mathrm{pH}$-sensitive self-assembly of spider silk proteins. J Phys Chem Lett 3:658-662. https://doi.org/10.1021/jz2016846

Warburg O (1925) über den Stoffwechsel der Carcinomzelle. Klinische Wochenschrift 4: 534-536. https://doi.org/10.1007/BF01726151

Warburg O (1956) On the origin of cancer cells. Science 123:309-314. https://doi.org/10.1126/science.123.3191.309

Ward C, Langdon SP, Mullen P, Harris AL, Harrison DJ, Supuran CT, Kunkler IH (2013) New strategies for targeting the hypoxic tumour microenvironment in breast cancer. Cancer Treat Rev 39:171-179. https://doi.org/10.1016/j.ctrv.2012.08.004

Warwicker J (2021) A model for $\mathrm{pH}$ coupling of the SARS-CoV-2 spike protein open/closed equilibrium. Brief Bioinform 22:14991507. https://doi.org/10.1093/bib/bbab056

Zhang X, Lin Y, Gillies RJ (2010) Tumor pH and its measurement. J Nucl Med 51:1167-1170. https://doi.org/10.2967/jnumed.109. 068981 